\title{
Mechanical Fabrication of Silver Cap onto Silica Nanotubes
}

\author{
Jaeeun Yu, ${ }^{\dagger+*}$ Won Jung Yoon, ${ }^{\S}$ and Sang Jun Son ${ }^{\dagger, *}$ \\ ${ }^{\dagger}$ College of BioNano Technology, Kyungwon University, Seongnam, Gyeonggi 461-701, Korea \\ *E-mail: sjson@kyungwon.ac.kr \\ ${ }^{\star}$ Department of Chemistry, Seoul National University, Seoul 151-747, Korea \\ ${ }^{\S}$ Department of Chemical and Bio Engineering, Kyungwon University, Seongnam, Gyeonggi 461-701, Korea \\ Received March 19, 2010, Accepted April 6, 2010
}

Key Words: Silica nanotubes, Mechanical capping, Hammering treatment, Silver-caps

Template-synthesized silica nanotubes (SNTs) have proven to be an ideal multifunctional nanostructure for the biomedical applications such as drug delivery and bioseparation. ${ }^{1-4}$ Nanotubes provide the advantage over nanospheres in that their inner voids can be used for loading large amounts of drug molecules and the differential functionalization of the inner and outer surfaces of SNTs enables appropriate surface modifications compatible with their environment. ${ }^{5-8}$ In addition, due to the tubular shape of nanotubes, the open end of SNTs can be capped to encapsulate cargo molecules and thus may control drug/gene uptake and release rate. ${ }^{9-11}$

There have been attempts to fabricate caps at the open end of SNTs. Martin and co-workers have published corking processes using the chemical reaction between amine-functionalized open ends of SNT and aldehyde-functionalized polymer nanoparticles. ${ }^{9}$ Another approach to cap NTs with gold nanoparticles was attempted by Lee and co-workers using pore diameter-dependent, self-regulating diffusion of Au NPs into the inside of SNTs, followed by seed-mediated Au growth reaction. ${ }^{10}$ Recently, we reported new capping strategy of SNTs involving thermal deposition of metal source and hammering treatment with alumina microbead. ${ }^{11}$ In contrast to these two previous works, the mechanical capping method can be achieved without surface modification or chemical reactions. Thus, chemical compatibility problems between cargo molecules and capping chemical reaction can be avoided. Another virtue of the mechanical capping method is that any material with appropriate malleability such as Au can be employed as the capping materials. In this paper, we report the systematic studies on the fabrication of Ag-capped SNTs under various conditions using the mechanical capping strategy.

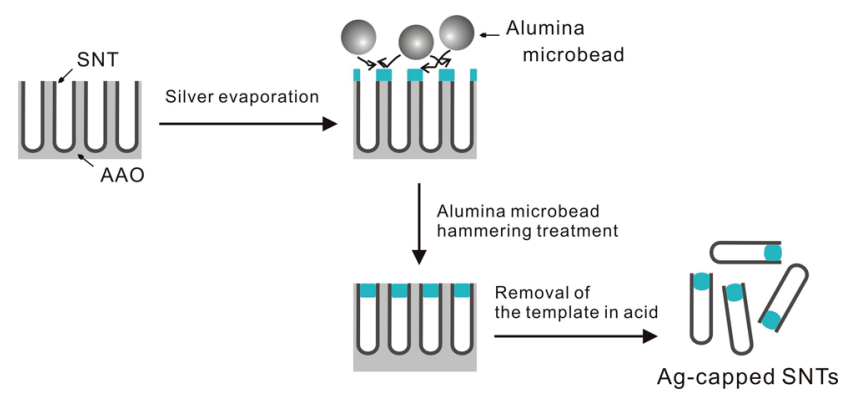

Figure 1. Schematic diagram for the preparation of Ag-cap using alumina microbead hammering treatment.
A schematic diagram for the preparation of Ag-capped SNTs was described in Fig. 1. First, SNTs were prepared in the pores of anodic aluminum oxide (AAO) template by a surface sol-gel (SSG) method. ${ }^{10,12}$ A silver layer (up to 80-nm thickness) was deposited using thermal evaporator and the resulting template was treated with alumina microbead hammering by shaking vigorously in a microtube vortex for up to $2 \mathrm{~d}$. Fig. 2 shows the SEM images of SNT-grown AAO with Ag layer on the top

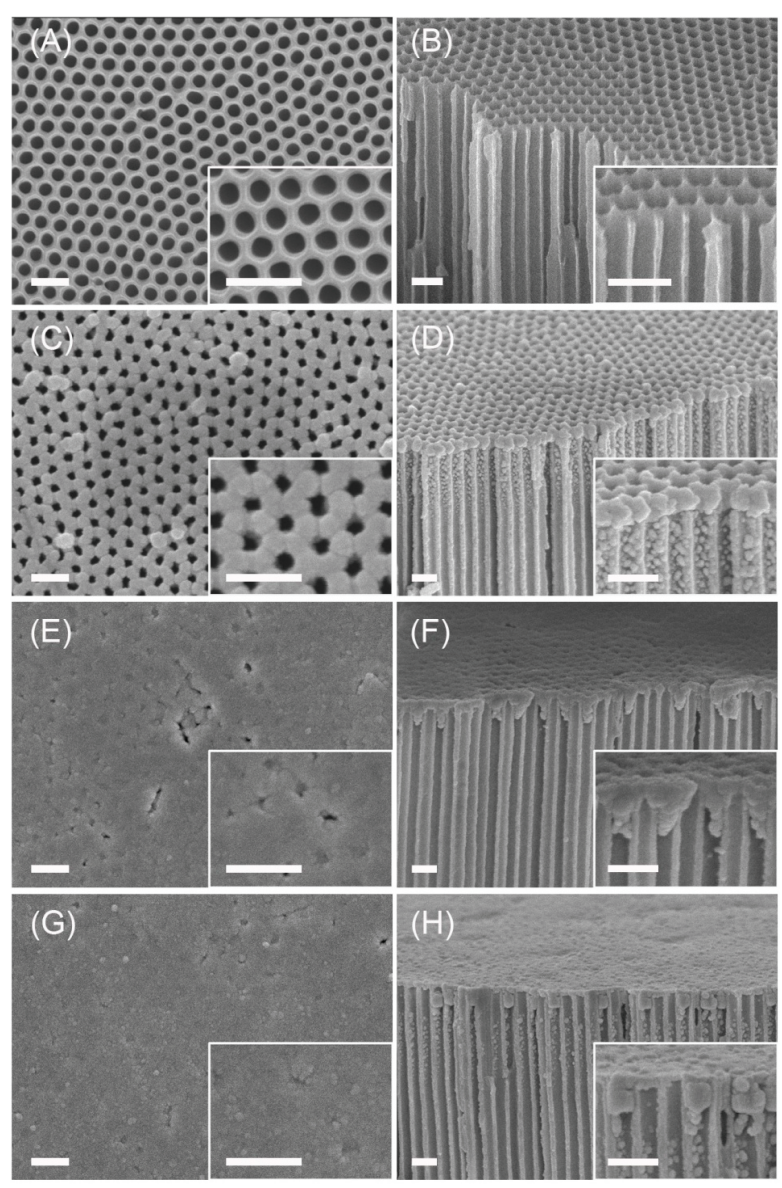

Figure 2. Top-viewed (A) and side-viewed (B) SEM images of AAO template of $6 \mu \mathrm{m}$ in length and $70 \mathrm{~nm}$ in diameter. $(\mathrm{C}-\mathrm{H})$ Top-viewed and side-viewed SEM images of SNT-grown AAO with 80-nm thickness of $\mathrm{Ag}$ layer after alumina beads hammering treatment for given times; (C, D) for $0 \mathrm{~min},(\mathrm{E}, \mathrm{F})$ for $6 \mathrm{hr}$, and $(\mathrm{G}, \mathrm{H})$ for $2 \mathrm{~d}$. Scale bars are $200 \mathrm{~nm}$. 
before and after alumina microbeads hammering treatment for the given times.

Ag-capping of SNTs can be achieved because silver is malleable like Au. Mechanical contact with the harder alumina microbeads enables the Ag layer to be inserted inside of SNT and to reform into the cylindrical cap shape. As seen in Fig. $2 \mathrm{C}$ and $2 \mathrm{D}$, although the pore diameter significantly decreases after the Ag deposition compared with the SNT-grown AAO template (Fig. 2A and 2B), the pores are still open. In contrast, after hammering treatment with alumina microbeads for the given time, most of the pores are closed (Fig. 2E-2H).

As the hammering treatment time increases, silver caps begin to be formed and the degree of pore closing depends on the microbead hammering treatment time. Since the pore diameter already decreased much after deposition of silver, there were a few pores remained open after only 10 min hammering with alumina microbeads. In addition, top-viewed (Fig. 2E) and sideviewed (Fig. 2F) SEM images of SNT-grown AAO after $6 \mathrm{~h}$ hammering treatment showed the completely Ag-covered template. However, silver caps were connected among themselves as seen in the side-viewed SEM images of this sample, indicating that more treatment with alumina microbeads is required to fabricate independent silver caps. After $2 \mathrm{~d}$ treatment, the SEM images (Fig. 2G and 2H) clearly show the silver caps formed at the open ends of nanotubes. Fig. 3 shows TEM images of free standing Ag-capped SNTs after dissolving the alumina template, which demonstrates that Ag-cap was successfully fabricated at the open end of the SNTs. The results support that the mechanical capping method is valid for the synthesis of various capping material with appropriate malleability.

To investigate the dependence of mechanical capping on the thickness of the Ag layer deposited initially onto the template, 6- $\mu \mathrm{m}$ SNT-grown AAO templates with different thickness of $\mathrm{Ag}$ layer $(30 \mathrm{~nm}, 60 \mathrm{~nm}$, and $90 \mathrm{~nm}$ ) were prepared and Agcapped SNTs liberated from each template were characterized by TEM. As anticipated, the thicker silver layer on the template produced the larger size of caps at the open ends part (Fig. 3B-
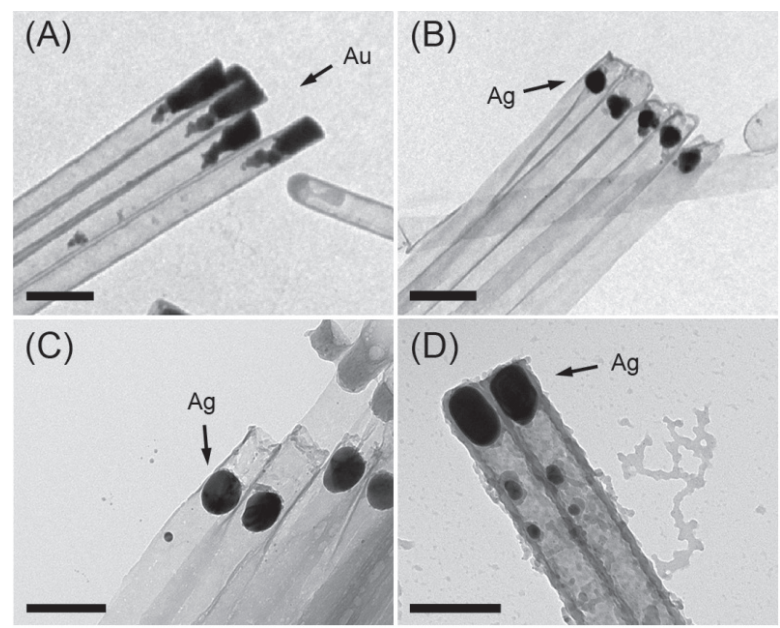

Figure 3. TEM images of Au-capped SNTs (A) and Ag-capped SNTs of $6 \mu \mathrm{m}$ in length and $70 \mathrm{~nm}$ in diameter (B-D); Ag-capped SNTs resulted from 30-nm (B), 60-nm (C), and 90-nm (D) thickness of Ag deposition, respectively. Scale bars are $200 \mathrm{~nm}$.
3D). This implies that the size of caps can be tuned by controlling the initial deposition thickness. However, in case of Agcapped SNTs prepared from 30-nm and 60-nm thickness of Ag layers (Fig. 3B and 3C), the silver caps seem not to be large enough to perfectly seal the open entrance part of nanotubes. Hence, in order to obtain SNTs completely capped with Ag, we can deduce that initial Ag deposition should be over $60 \mathrm{~nm}$ in thickness.

Compared with Au cap (Fig. 3A), the rounded cylindrical shape of Ag cap is noticeable. Nano-sized Ag is known to be susceptible to oxidation and the degradation problem in aqueous solution have been reported. ${ }^{13,14}$ Although the degradation of $\mathrm{Ag}$ could be one reason for the rounded shape of $\mathrm{Ag}$ cap, it is more likely that the relatively lower chemical stability of Ag to acidic condition (phosphoric acid used for the dissolution of AAO template) led to this shape change. However, drastic changes in the silver caps were not observed even after incubation in aqueous solution for 11 weeks.

To evaluate capping ability of Ag cap that can prohibit release of cargo molecules from inside of the SNT, dye release test with fluorescein-encapsulated Ag-capped SNTs was performed while the SNTs were embedded in the pores of the template. Before deposition of silver, SNT-grown AAO was immersed in a dye solution and dried in the air under darkness. The same processes as showed in Fig. 1 were then performed. Three samesized $\left(0.72 \mathrm{~cm}^{2}\right)$ SNT-grown templates were prepared with different hammering times $(0,10 \mathrm{~min}, 2 \mathrm{~d})$. Fig. 4A shows that the amounts of fluorescein released from the inside of SNTs decreased with the increase of hammering time. The amount of fluorescein in each solution was calculated using fluorescence standard curve of fluorescein in 1X PBS. The amounts of released molecules from the given size of templates were 18.5 nmol for $0 \mathrm{~min}, 9.23 \mathrm{nmol}$ for $10 \mathrm{~min}$, and $3.18 \mathrm{nmol}$ for $2 \mathrm{~d}$. Assuming that the maximum amount of fluorescein equals the amount of fluorescein released from the SNTs without the hammering treatment, the total percent of loaded fluorescein that were released was $50.0 \%$ and $17.2 \%$, respectively. Inversely, the amount of encapsulated fluorescein was also calculated based on the amount of fluorescein released from 0 min hammering-treated template. The amounts of encapsulated fluorescein inside nanotubes were $9.24 \mathrm{nmol}$ for $10 \mathrm{~min}$ and $15.3 \mathrm{nmol}$ for $2 \mathrm{~d}$. Thus, the percent encapsulation efficiency of Ag-capped SNTs was obtained as $50.0 \%$ and $82.8 \%$, respectively (Fig. 4B).

Our mechanical capping method turned out to be very useful for the encapsulation of cargo molecules because loaded molecules do not need to be functionalized in order to be immobilized onto the surface of nanomaterials. ${ }^{11}$ Fig. $4 \mathrm{C}$ shows bright field optical microscopy images of free standing Ag-capped SNTs after the release test and removal of the AAO template. Although the fluorescein is still encapsulated inside of SNTs, fluoresceinencapsulated SNTs with Ag caps, however, did not exhibit strong green fluorescence (Fig. 4D). We reason that the reduced fluorescence intensity is mainly caused by fluorescence quenching effect by relatively huge Ag caps ( $c a$. 100-nm nanoparticles). ${ }^{15}$ These results demonstrates that the encapsulation ability of Ag caps is apparent and can be used to encapsulate other functional molecules compatible with the Ag caps, such as MR contrasting agent and NIR imaging agent. 

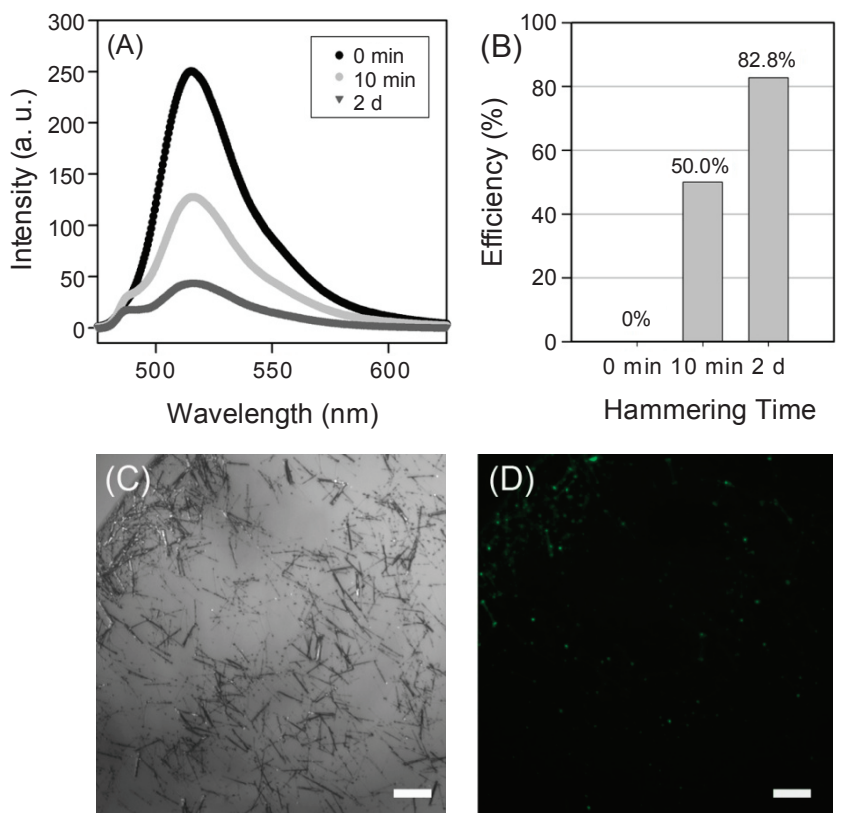

Figure 4. (A) Fluorescence spectrum of released fluorescein from 6$\mu \mathrm{m}$ Ag-capped SNTs embedded in pores of the template treated with alumina microbeads hammering for given times; $0 \mathrm{~min}(\bullet), 10 \mathrm{~min}(\mathrm{O})$, and $2 \mathrm{~d}(\boldsymbol{\nabla})$. These solutions were diluted ten times for measurements. (B) Capping efficiency of Ag-capped SNTs depending on alumina microbead hammering treatment times. Bright-field optical microscopy (C) and fluorescence microscopy (D) of fluorescein-encapsulated Ag-capped fluorescent SNTs of $6 \mu \mathrm{m}$ in length. Scale bars are $10 \mu \mathrm{m}$.

In summary, we successfully synthesized Ag-capped SNTs using mechanical capping method and systematically investigated Ag cap fabrication under various conditions. The capping ability of Ag cap was evaluated using fluorescence dye release test and encapsulation of cargo molecules into SNT was also demonstrated. The results support that mechanical capping method can serve as a platform technique for the synthesis of multifunctional SNTs capped with various capping material by encapsulating functional cargo molecules.

\section{Experimental Section}

Materials. Silicon tetrachloride $\left(\mathrm{SiCl}_{4}, 99.8 \%\right.$, Acros Organics), aluminum oxide (fused, -325 mesh, +10 micron, $99 \%$, Aldrich), silver (99.99\%, Kurt J. Lesker), fluorescein (free acid, 95\%, Aldrich), and phosphate buffered saline (PBS, pH 7.4, Sigma) were used as supplied without further purification. Aluminum foils $(99.99 \%$ ) were purchased from Alfa Aesar.

Preparation of the Ag-capped SNT. Alumina template was prepared according to the literature. ${ }^{16,17}$ SNTs were prepared using surface sol-gel method described previously. ${ }^{10,12}$ To obtain SNT with $10 \mathrm{~nm}$ wall thickness, 7 cycles of surface sol-gel was performed. Ag-capped SNTs were prepared by alumina microbead hammering treatment. ${ }^{11}$ Briefly, a Ag layer was thermally deposited onto the SNT-grown AAO templates with a growth rate of $0.20 \mathrm{~nm} / \mathrm{s}$. Hammering treatments was performed by shaking with commercial alumina powder for 2 days. The process of silver capping was characterized by a field emission scanning electron microscope (FE-SEM, JEOL JSM-6700F). Free-standing capped-SNTs of $6 \mu \mathrm{m}$ in length and $70 \mathrm{~nm}$ in diameter were obtained after dissolution of the alumina template with phosphoric acid solution ( $25 \mathrm{wt} \%$ ) for a day. The obtained nanotubes were characterized by an energy-filtering transmission electron microscope (EF-TEM, Zeiss LIBRA 120) and a fluorescence microscope (Nikon ECLIPSE 80i).

Release study of fluorescein from SNTs. To a solution of fluorescein $\left(6.5 \mathrm{mM}\right.$, in methanol), three same-sized $\left(0.75 \mathrm{~cm}^{2}\right)$ SNT-grown templates of $6 \mu \mathrm{m}$ in length were immersed for $1 \mathrm{~min}$ and dried under darkness, then the templates were treated with different hammering times $(0,10 \mathrm{~min}, 2 \mathrm{~d})$. The resultant templates were incubated with $1 \mathrm{~mL}$ of $1 \mathrm{X}$ PBS buffer solution for an hour, twice. Both the solutions were combined together and fluorescence intensity of the solution was measured by using luminescence spectrometer (Perkin-Elmer LS55). Excitation wavelength was $494 \mathrm{~nm}$ and emission values at $516 \mathrm{~nm}$ were used for further calculation. Excitation and emission slit widths were $15 \mathrm{~nm}$ and $5 \mathrm{~nm}$, respectively.

Acknowledgments. This work was supported by the Korea Research Foundation Grant funded by the Korean Government (KRF-2008-313-C00581). This research was supported by the Kyungwon University Research Fund in 2007.

\section{References}

1. Mitchell, D. T.; Lee, S. B.; Trofin, L.; Li, N. C.; Nevanen, T. K.; Soderlund, H.; Martin, C. R. J. Am. Chem. Soc. 2002, 124, 1186411865 .

2. (a) He, B.; Son, S. J.; Lee, S. B. Langmuir 2006, 22, 8263-8265. (b) He, B.; Son, S. J.; Lee, S. B. Anal. Chem. 2007, 79, 5257-5263.

3. Nan, A.; Bai, X.; Son, S. J.; Lee, S. B.; Ghandehari, H. Nano Lett. 2008, 8, 2150-2154.

4. Buyukserin, F.; Medley, C. D.; Mota, M. O.; Kececi, K.; Rogers, R. R.; Tan, W.; Martin, C. R. Nanomed. 2008, 3, 283-292.

5. Chen, C.-C.; Liu, Y.-C.; Wu, C.-H.; Yeh, C.-C.; Su, M.-T.; Wu, Y.-C. Adv. Mater. 2005, 17, 404-407.

6. (a) Son, S. J.; Reichel, J.; He, B.; Schuchman, M.; Lee, S. B. J. Am. Chem. Soc. 2005, 127, 7316-7317. (b) Bai, X.; Son, S. J.; Zhang, S.; Liu, W.; Jordan, E. K.; Frank, J. A.; Venkatesan, T.; Bok Lee, S. Nanomed. 2008, 3, 163-174.

7. Son, S. J.; Bai, X.; Nan, A.; Ghandehari, H.; Lee, S. B. J. Control. Release 2006, 114, 143-152.

8. Hillebrenner, H.; Buyukserin, F.; Stewart, J. D.; Martin, C. R. Nanomed. 2006, 1, 39-50.

9. Hillebrenner, H.; Buyukserin, F.; Kang, M.; Mota, M. O.; Stewart, J. D.; Martin, C. R. J. Am. Chem. Soc. 2006, 128, 4236-4237.

10. Son, S. J.; Lee, S. B. J. Am. Chem. Soc. 2006, 128, 15974-15975.

11. Yu, J.; Bai, X.; Suh, J.; Lee, S. B.; Son, S. J. J. Am. Chem. Soc. 2009, $131,15574-15575$.

12. Kovtyukhova, N. I.; Mallouk, T. E.; Mayer, T. S. Adv. Mater. 2003, 15, 780-785.

13. Nicewarner-Pena, S. R.; Carado, A. J.; Shale, K. E.; Keating, C. D. J. Phys. Chem. B 2003, 107, 7360-7367.

14. Stoermer, R. L.; Sioss, J. A.; Keating, C. D. Chem. Mater. 2005, 17, 4356-4361.

15. Castanie, E.; Boffety, M.; Carminati, R. Optics Lett. 2010, 35, 291-293.

16. Masuda, H.; Fukuda, K. Science 1995, 268, 1466-1468.

17. Li, A. P.; Muller, F.; Birner, A.; Nielsch, K.; Gosele, U. J. Appl. Phys. 1998, 84, 6023-6026. 